\title{
Photoinduced Charge Carrier Generation in a Poly(3-hexylthiophene) and Methanofullerene Bulk Heterojunction Investigated by Time-Resolved Terahertz Spectroscopy ${ }^{\dagger}$
}

\author{
Xin Ai, Matthew C. Beard,* Kelly P. Knutsen, Sean E. Shaheen, Garry Rumbles, and \\ Randy J. Ellingson \\ National Renewable Energy Laboratory, 1617 Cole Boulevard, Golden, Colorado 80401
}

Received: August 11, 2006; In Final Form: October 4, 2006

\begin{abstract}
We report on the ultrafast photoinduced charge separation processes in varying compositions of poly(3hexylthiophene) (P3HT) blended with the electron acceptor [6,6]-phenyl- $\mathrm{C}_{61}$-butyric acid methyl ester (PCBM). Through the use of time-resolved terahertz spectroscopy, the time- and frequency-dependent complex photoconductivity is measured for samples with PCBM weight fractions ( $\left.W_{\mathrm{PCBM}}\right)$ of $0,0.2,0.5$, and 0.8 . By analysis of the frequency-dependent complex conductivity, both the charge carrier yield and the average charge carrier mobility have been determined analytically and indicate a short $(<0.2 \mathrm{~nm})$ carrier mean free path and a suppressed long-range transport that is characteristic of carrier localization. Studies on pure films of P3HT demonstrate that charge carrier generation is an intrinsic feature of the polymer that occurs on the time scale of the excitation light, and this is attributed to the dissociation of bound polaron pairs that reside on adjacent polymer chains due to interchain charge transfer. Both interchain and interfacial charge transfer contribute to the measured photoconductivity from the blended samples; interfacial charge transfer increases as a function of increasing PCBM. The addition of PCBM to the polymer films surprisingly does not dramatically increase the production of charge carriers within the first 2 ps. However, charge carriers in the 0.2 and 0.5 blended films survive to much longer times than those in the P3HT and 0.8 films.
\end{abstract}

\section{Introduction}

Organic solar cells based on the bulk heterojunction of conjugated polymer/fullerene compounds have been intensely studied recently as a promising low-cost replacement for conventional silicon photovoltaics. Using conjugated polymer/ fullerene composites has numerous advantages, such as low toxicity, adjustable electronic or mechanical properties through functionalization, and ease of fabrication. Highly efficient solar cells based on a donor-type polymer regioregular poly(3hexylthiophene) (P3HT) and acceptor [6,6]-phenyl- $\mathrm{C}_{61}$-butyric acid methyl ester (PCBM) bulk heterojunction have been reported, ${ }^{1-5}$ achieving a power-conversion efficiency as high as $4.4 \%{ }^{5}$ Higher efficiencies have been reported but not certified. ${ }^{6,7}$ Many factors influence device performance, including the regioregularity of $\mathrm{P} 3 \mathrm{HT},{ }^{8}$ composition of the blended film, ${ }^{2,3,9}$ solvent, ${ }^{10}$ film thickness, ${ }^{11}$ annealing temperature, ${ }^{4,9}$ and annealing time. ${ }^{11}$ Among these factors, the precise composition of the blended films plays a critical role in determining the performance of the cell. For example, a P3HT/PCBM weight ratio of $1: 1$ tends to perform better than other ratios. ${ }^{2,3,9}$ However, the reason for this is not clear. A systematic study of the interfacial interaction between P3HT and PCBM including microscopic charge generation, separation, migration, and collection as functions of varying composition will advance understanding of their roles in determining device performance.

Several experimental techniques have been used to investigate how varying the composition affects device performance. Atomic force microscopy reveals the role of morphology in the blend: The growth of PCBM clusters build up percolation paths,

\footnotetext{
† Part of the special issue "Arthur J. Nozik Festschrift".

* Author to whom correspondence should be addressed. E-mail: matt_beard@nrel.gov.
}

which improves the collection of photocurrent. However, above a certain PCBM concentration, the PCBM crystals may exert too much mechanical stress on the metal electrode and thereby damage the interfacial connection. ${ }^{2}$ Huang et al. ${ }^{12}$ utilized the time-of-flight technique to investigate the influence of composition on the transport properties in P3HT/PCBM cells and found that for both electrons and holes transport shows a transition from dispersive to nondispersive and back to dispersive again with increasing PCBM content in the blend. Time-resolved microwave conductivity (TRMC), another powerful technique to study the mobility of charge carriers in conjugated polymers, has been utilized to study the composition effect in poly[2methoxy-5-(3',7'-dimethyloctylosy)-p-phenylene vinylene] (MDMO-PPV) and PCBM blended films. ${ }^{13}$ The TRMC results show that the photoconductivity reaches a maximum value for a weight fraction of $W_{\text {PCBM }}=0.85$ probably due to phase separation and formation of highly mobile electrons within PCBM-rich aggregates. These studies provide insight into carrier relaxation and recombination processes at nanosecond to millisecond time scales. However, the effect of blend composition on the interfacial charge carrier generation, which generally occurs on the sub-picosecond to picosecond time scale, ${ }^{14,15}$ is still not well understood.

In recent years, time-resolved terahertz spectroscopy (TRTS), ${ }^{16}$ a noncontact probe of the photoconductivity with subpicosecond to nanosecond temporal resolution, has been successfully applied to measurements of the photoconductivity in conjugated polymer systems. ${ }^{17-21}$ TRTS is a unique experimental probe that allows for the determination of the photonto-carrier generation efficiency because it is sensitive to the charge carriers (or charged species called polarons in conducting polymer) and can probe on time scales short enough to resolve ultrafast carrier generation dynamics. Meanwhile, tightly bound 
excitons-electron-hole pairs on a single polymer chain generated from intrachain photoexcitation-contribute to the TRTS response at a much reduced level compared to charge carriers. Furthermore, as discussed below, excitons exhibit a distinct frequency dependence compared to that of charge carriers. ${ }^{21}$ Therefore, it is also possible to distinguish charge carriers from excitons in the TRTS response. There has been much discussion about how charge carriers are generated and with what yield within photoconductive polymers. A pioneering study by Hendry et al. ${ }^{21}$ utilized TRTS measurements to confirm that photoexcitation produces only a very small fraction of charge carriers on the sub-picosecond time scale in pure poly[2-methoxy-5(2'ethyl-hexyloxy)-1,4-phenylene vinylene] (MEH-PPV) films. The photon-to-carrier conversion efficiency for MEH-PPV films was measured to be $\sim 10^{-3}$.

In this paper, the photoinduced charge carrier generation dynamics of P3HT/PCBM blended films with PCBM weight fractions of $0,0.2,0.5$, and 0.8 are studied by TRTS. The samples were excited by $500 \mathrm{~nm}$ light, and the differential transmitted far-infrared electric field is measured as a function of temporal delay, $\tau_{\mathrm{d}}$, between the visible pump and the terahertz probe pulses. By comparison of the differential transmitted field to the transmitted field strength without a pump, the timeresolved frequency-dependent photoconductivity is extracted and used to reveal the origin of the signal in the blended films with sub-picosecond resolution.

\section{Experimental Section}

PCBM/P3HT Blend Film preparation. The fullerene derivative PCBM (NanoC) and regioregular P3HT (Merck, average molecular weight, $M_{\mathrm{w}}=21.2 \mathrm{~kg} / \mathrm{mol}$, number average $M_{\mathrm{n}}=$ $10.6 \mathrm{~kg} / \mathrm{mol}, 93.7 \%$ regioregular) were used as received. A series of PCBM/P3HT chloroform solutions with total active material concentrations of $15 \mathrm{mg} / \mathrm{mL}$ with PCBM weight fractions of $W_{\mathrm{PCBM}}=0,0.2,0.5,0.8$, and 1.0 were prepared and stirred at $70{ }^{\circ} \mathrm{C}$ overnight. The blended solutions were spin-coated in air on precleaned glass substrates to measure the absorption coefficient. The film thicknesses were measured by a Dektak profilometer to be $158,113,105,84$, and $94 \mathrm{~nm}$ for $W_{\mathrm{PCBM}}=$ $0,0.2,0.5,0.8$, and 1.0 films, respectively. A second set of thicker films was prepared for the terahertz experiments where an optical density (OD) greater than 2.0 is desired to ensure that each film absorbs $99 \%$ of the pump light. To produce these thicker films, solutions were drop-cast onto clean Z-cut quartz substrates and subsequently dried in air; the drying time was approximately $2 \mathrm{~min}$. The film samples are referred to as P3HT, $0.2,0.5,0.8$, and PCBM films respectively. The P3HT, 0.2, and 0.5 blends form relatively homogeneous films; films cast from 0.8 and PCBM show inhomogeneity. The average thicknesses are 5.3, 13, 5.0, 1.8, and $0.7 \mu \mathrm{m}$ for P3HT, 0.2, 0.5, 0.8, and PCBM films, respectively.

Terahertz Spectroscopy Setup and Experiment. The setup for TRTS measurements is similar to that reported previously. ${ }^{22}$ The fundamental laser source for terahertz generation and detection as well as for generation of the pump light, is a 1 kHz, 60-fs, 810-nm, amplified Ti:sapphire laser (Quantronix, Integra-E). To generate the terahertz probe beam, approximately $400 \mu \mathrm{J} /$ pulse of $810 \mathrm{~nm}$ collimated light with a spot size of 8 $\mathrm{mm}$ in diameter impinges on a 1-mm-thick $\langle 110\rangle$ ZnTe crystal. The terahertz light is focused onto the sample with an off-axis parabolic mirror, and the transmitted terahertz light is recollimated and then focused onto another 1-mm-thick $\langle 110\rangle \mathrm{ZnTe}$ crystal for detection. A small part of the fundamental $810 \mathrm{~nm}$ light, approximately $10 \mathrm{~nJ} /$ pulse, is focused onto the ZnTe detector crystal and spatially overlapped with the terahertz pulse. Reflection of the $810 \mathrm{~nm}$ gate pulse in the ZnTe crystal is used to couple the $810 \mathrm{~nm}$ light into the detector crystal collinearly with the terahertz pulse. The terahertz pulse is detected via freespace electro-optic sampling; ${ }^{23,24}$ unbiased, balanced photodiodes are used to collect the gating detector light. The pump light, at $500 \mathrm{~nm}$, is generated from an optical parametric amplifier (OPA, Topas) and typically has a beam diameter of 3 $\mathrm{mm}$ at the sample, slightly larger than that of the terahertz pulse.

Two variants of TRTS experiments can be employed, both of which are subsets of the full experimental capability. ${ }^{22,25} \mathrm{In}$ one variant, the change in terahertz field transmission $(\Delta E / E)$ is measured at a point, or particular delay, on the terahertz waveform as a function of varying pump-probe delay $\left(\tau_{\mathrm{d}}\right)$. In the second variant, the full terahertz waveform is measured, scanning all the terahertz delays $(t)$ at a fixed pump delay. In the first experiment, dynamical information is obtained. The overall magnitude of $\Delta E / E$, measured at the point in time $\left(t_{\max }\right)$ where the differential signal is maximum, is proportional to the product of the carrier density, $N$, and mobility, $\mu$. In the second experiment, the frequency-dependent terahertz response is obtained at a particular pump delay, allowing for modeling of the carrier transport mechanisms to separate $N$ and $\mu$. One of the attractive features of TRTS is the possibility of extracting $N$ and $\mu$ separately in one experiment.

Let us consider the measured $\Delta E / E$ : In the plane wave approximation, the electric field at time $t$ and position $z$ in one dimension is given by $E(z, t)=E_{0} \exp (\mathrm{i} \tilde{\kappa} z-\mathrm{i} \omega t)$ where $E_{0}$ is the field envelope, $\omega$ is its carrier frequency, and $\tilde{\kappa}(\omega)$ is the wavevector, which is related to the optical constants by $\tilde{\kappa}(\omega)$ $=\omega n(\omega) / c+i \alpha(\omega) / 2$, where $n(\omega)$ is the refractive index and $\alpha(\omega)$ is the absorption coefficient. These parameters are also related to the generalized permittivity, $\tilde{\eta}(\omega)$, by $\sqrt{\eta(\omega)}=$ $c \tilde{\kappa}(\omega) / \omega$. The permittivity can be separated into contributions from both bound and unbound charges, $\tilde{\eta}=\tilde{\epsilon}+\mathrm{i} \tilde{\sigma} / \omega \epsilon_{0}$, where $\tilde{\epsilon}$ is the frequency-dependent complex dielectric constant associated with bound charges, $\epsilon_{0}$ is the permittivity of free space, and $\tilde{\sigma}$ is the complex frequency-dependent conductivity (the explicit frequency dependence has been omitted) associated with unbound charges. Assuming that photoexcitation only produces changes in $\sigma, \Delta E / E$ can be related to the average photoconductivity (averaged over the probe frequency spectrum) by

$$
\frac{\Delta E\left(\tau_{\mathrm{d}}, t_{\max }\right)}{E\left(t_{\max }\right)} \cong-\frac{\sigma\left(\tau_{\mathrm{d}}\right) z}{2 \epsilon_{0} c n}=-\frac{e \mu(\tau) N\left(\tau_{\mathrm{d}}\right) z}{2 \epsilon_{0} c n}
$$

where $z$ is the thickness of the photoexcited material. Therefore, a decrease in the absolute value of $\Delta E / E$ may result from either a decrease in the carrier density, $N\left(\tau_{\mathrm{d}}\right)$, or a decrease in the mobility, $\mu\left(\tau_{\mathrm{d}}\right)$. Only by extracting the full frequency-dependent complex conductivity at each delay is it possible to distinguish between a decrease in carrier density and/or a decrease in mobility.

Therefore, we need to extract the frequency-dependent complex conductivity from the measured $\Delta E(t) / E(t)$. One is tempted to write from eq $1^{19}$

$$
\frac{\Delta \tilde{E}\left(\omega ; \tau_{\mathrm{d}}\right)}{\tilde{E}(\omega)} \approx-\frac{\tilde{\sigma}\left(\omega ; \tau_{\mathrm{d}}\right) z}{2 \epsilon_{0} c n}
$$

where the electric field and conductivity are complex. However, this is only true for very small $\Delta E / E$ values. To extract the most accurate frequency-dependent complex conductivity, we use the measured $E_{\mathrm{ph}}^{*}\left(\omega, \tau_{\mathrm{d}}\right) / E(\omega)$, where $E_{\mathrm{ph}}^{*}=\Delta E+E$ is the photo- 

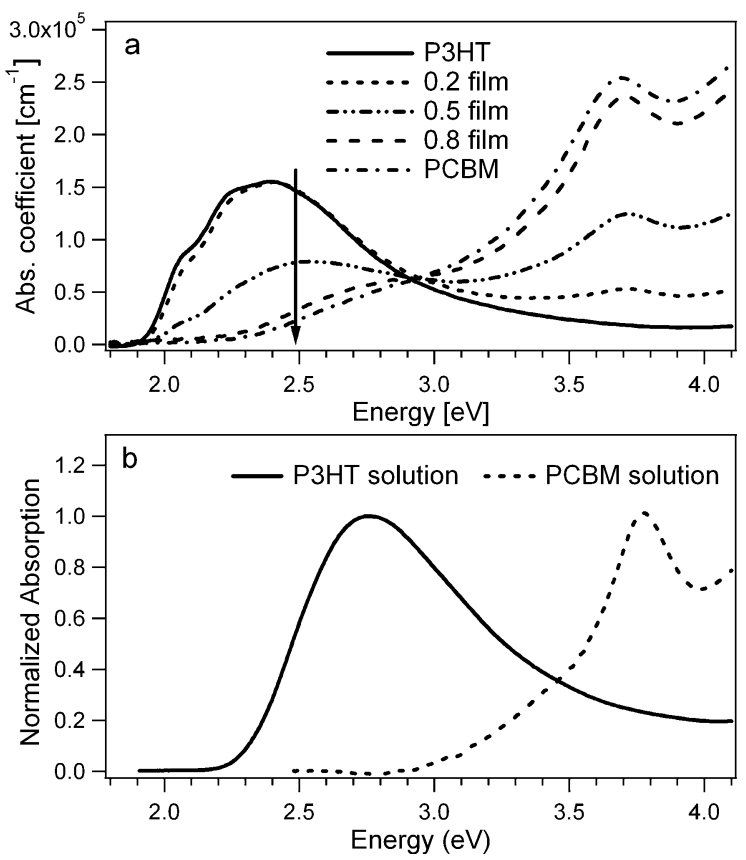

Figure 1. (a) Absorption coefficients of PCBM/P3HT blended films with $W_{\text {PCBM }}=0,0.2,0.5,0.8$, and 1.0 respectively (see legend). For TRTS measurements, $500 \mathrm{~nm}$ light is used to excite the films (indicated by the arrow). (b) Normalized absorption spectra of pure P3HT and PCBM diluted chloroform solutions.

excited transmitted field amplitude recorded at a pump delay $\tau_{\mathrm{d}}$; this ratio is the complex transmission coefficient, which has been defined in the supplemental information of a previous publication. ${ }^{26}$ We then model the sample as a dielectric stack with four layers - air|photoexcited material|non-photoexcited material|quartz substrate- and use the procedure developed by Beard et al. ${ }^{22,26}$ to extract the measured complex conductivity. We need to know the thickness of each layer and the static farIR optical constants for each layer except the unknown photoexcited material. The thickness of the photoexcited layer, $\delta$, is determined from the absorption coefficient (see below), $\delta$ $=1 / \alpha$, at $500 \mathrm{~nm}$. We assume that the photoexcited material is a uniformly excited slab of thickness $\delta$, while in reality it is an exponentially decreasing distribution with a $1 / e$ value of $\delta$. For this P3HT/PCBM system, $\delta=70,70,125$, and $300 \mathrm{~nm}$ for the P3HT, 0.2, 0.5, and 0.8 films respectively, which are much smaller than the wavelength of light $(\lambda=300 \mu \mathrm{m}$ at $1 \mathrm{THz})$; therefore it is appropriate to use the slab approximation as noted in the supplemental information discussed earlier. ${ }^{26}$ The nonphotoexcited far-IR optical properties are determined in a separate static terahertz measurement. We find that the nonphotoexcited films do not absorb terahertz radiation, and therefore, only the refractive index, $n$, of each film was measured. The average refractive index of each film is approximately the volume-weighted average of pure P3HT, $n_{\mathrm{P} 3 \mathrm{HT}}$ $=2.22$, and PCBM, $n_{\mathrm{PCBM}}=1.35$. Comparing the extracted $\tilde{\sigma}(\omega)$ using this procedure to that of eq 2 yielded similar results that diverged for larger $\Delta E / E$ values, showing the shortfalls of applying the approximation in eq 2 . The measured frequencydependent complex conductivity, obtained independent of any model, is sensitive to the carrier transport mechanism.

\section{Results}

UV-Vis Spectra of P3HT, PCBM, and Their Blended Films. Figure 1a shows the absorption spectra of PCBM/P3HT blended films with differing weight ratios of the two compo- nents. As a reference, the normalized absorption spectra of pure P3HT and PCBM diluted in chloroform solutions are also shown in Figure 1b. In comparison to the P3HT solution spectrum (peaked at $\sim 2.76 \mathrm{eV}$ ), the absorption peak of the P3HT film is red-shifted to $\sim 2.40 \mathrm{eV}$, with two shoulders at approximately 2.25 and $2.07 \mathrm{eV}$. These three peaks correspond to the $\pi-\pi^{*}$ transition of $\mathrm{P} 3 \mathrm{HT}$, and their positions are consistent with reported observations. ${ }^{3}$ The red-shifted absorption of P3HT can be ascribed to more delocalized conjugated $\pi$ electrons due to increased ordering of the P3HT chains in the pure P3HT film. ${ }^{9}$ There are no new peaks in the absorption spectra of the blended films and therefore no evidence for a ground state electronic interaction between the P3HT and the PCBM constituents. The 0.2 film has a small PCBM absorption peak at $\sim 3.70 \mathrm{eV}$ but virtually retains the $\mathrm{P} 3 \mathrm{HT}$ film absorption features (in both shape and intensity), implying that the ordering of the polymer chains is not interrupted. For the 0.5 film, the P3HT absorption peak is blue-shifted from 2.4 to $\sim 2.53 \mathrm{eV}$ but retains the shoulders on the red edge. These observations indicate that while the P3HT molecules retain some short-range ordering in the 0.5 film this concentration of PCBM is beginning to disrupt the long-range ordering. Finally, the 0.8 film shows no sign of an ordered P3HT structure. Subtraction of the pure PCBM spectrum from the 0.8 spectrum results in a $\mathrm{P} 3 \mathrm{HT}$ absorption spectrum that peaks around $\sim 2.67 \mathrm{eV}$, only slightly red-shifted from the absorption of the P3HT solution. This indicates the destruction of both long- and short-range ordering in the P3HT chains by the presence of PCBM aggregates. Several other groups have reported that the absorption spectra of P3HT/PCBM blended thin films blue-shifted significantly when the amount of PCBM is $67 \mathrm{wt} \%$ or more, ${ }^{2,9}$ consistent with our observations.

Terahertz Dynamics. The average photoconductivity $\sigma\left(\tau_{\mathrm{d}}\right)$ of the P3HT, 0.2, 0.5, and 0.8 films as a function of delay time $\tau_{\mathrm{d}}$ between the $500 \mathrm{~nm}$ pump and terahertz probe pulses is shown in Figure 2. The average photoconductivity is obtained from $\Delta E\left(t_{\max }, \tau_{\mathrm{d}}\right) / E\left(t_{\max }\right)$ by eq 1 . The excitation density was $\sim 470 \mu \mathrm{J} / \mathrm{cm}^{2}$, corresponding to $\sim 1.2 \times 10^{15}$ photons $/ \mathrm{cm}^{2}$. For the pure PCBM film, an instantaneous signal was observed, but it decays to zero within 20 ps (data not shown). In Figure 2, all traces have an instantaneous rise of $\sim 0.4$ ps (limited by the instrument response) and nonexponential decays. The traces were fit by biexponential or triexponential decay functions convoluted with instrument response. The fits are shown as lines in Figure 2, and the fitting parameters are listed in Table 1. The signal amplitude at pump delay $\tau_{\mathrm{d}}=0.5 \mathrm{ps}$ decreases from the P3HT to 0.8 films as the PCBM content increases. However, the majority of the photoconductivity for the P3HT film decays within a picosecond, and only a small long-lived component remains. In contrast, the blended films do not display a large picosecond decay. The 0.8 film decays faster than the 0.2 and 0.5 films, the latter two showing similar biexponential (fast and slow) decay dynamics. As shown in Figure 2b, the photoconductivity remaining at 150 ps delay varies for the films as follows; the 0.2 film is largest, followed by the $0.5, \mathrm{P} 3 \mathrm{HT}$, and 0.8 films.

Frequency-Dependent Conductivity. TRTS is sensitive to the real and imaginary components of the frequency-dependent photoconductivity and, as such, can be a powerful tool to monitor free and bound charge dynamics. ${ }^{19-21}$ Figure 3a displays the differential transmitted electric field, $\Delta E\left(t, \tau_{\mathrm{d}}=5.0 \mathrm{ps}\right)$, for the 0.5 film at a $5 \mathrm{ps}$ pump delay time. The $\Delta E$ trace shows behavior characteristic of a conductive film: It is shifted forward in time but is otherwise a mirror image of the reference pulse. ${ }^{22,27}$ Figure $3 b$ displays the real, $\operatorname{Re}[\tilde{\sigma}(\omega)]$, and imaginary, 

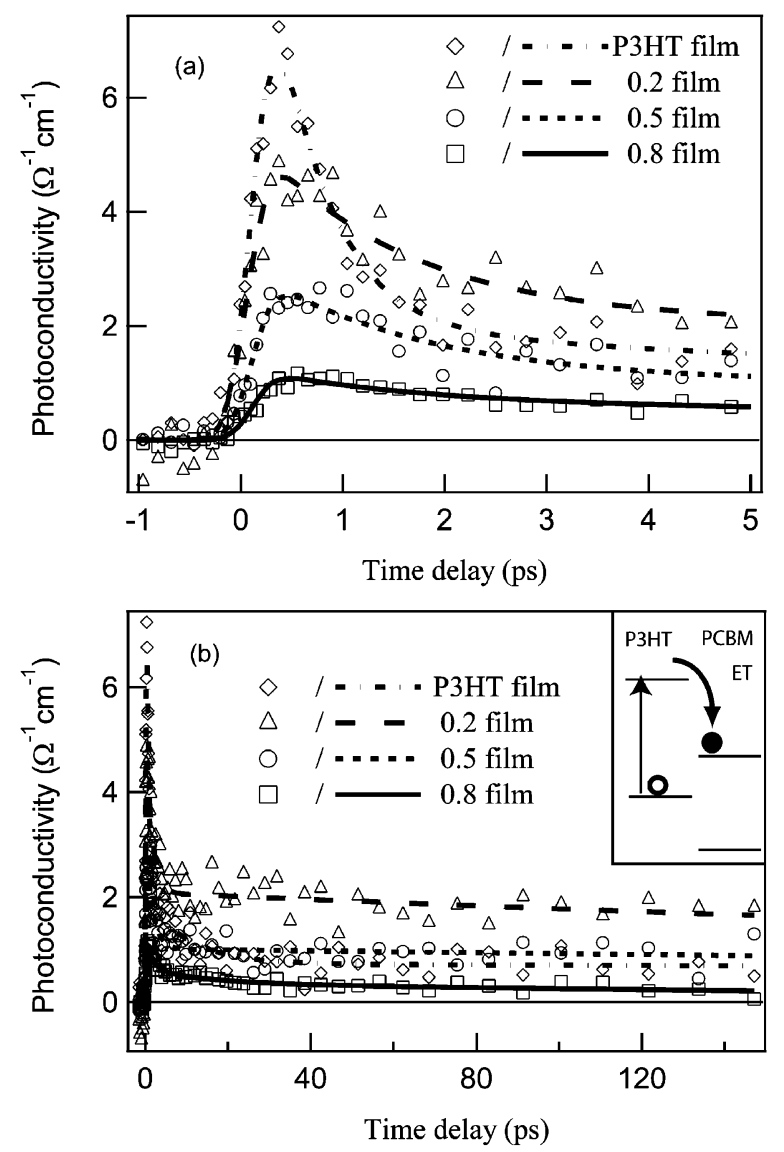

Figure 2. TRTS dynamic scans for P3HT, 0.2, 0.5, and 0.8 films at (a) early and (b) later delay times. Open symbols are raw data, and lines are biexponential or triexponential fits (see text). The inset in part $b$ shows the band offsets of P3HT and PCBM. Rapid electron transfer occurs after photoexcitation of the $\mathrm{P} 3 \mathrm{HT}$, producing electrons in the PCBM and holes in the P3HT.

TABLE 1: Bi- or Triexponential Fitting Parameters for TRTS Decay Dynamics of P3HT, 0.2, 0.5, and 0.8 Films Shown in Figure 2

\begin{tabular}{lcccccc}
\hline & $A_{1}(\%)$ & $\tau_{1}(\mathrm{ps})$ & $\mathrm{A}_{2}(\%)$ & $\tau_{2}(\mathrm{ps})$ & $A_{3}(\%)$ & $\tau_{3}(\mathrm{ps})$ \\
\hline P3HT & 80.7 & 0.55 & 12.3 & 10 & 7.0 & 2817 \\
0.2 film & 61.4 & 1.48 & 38.6 & 506 & & \\
0.5 film & 65.8 & 1.70 & 34.2 & 448 & & \\
0.8 film & 48.7 & 1.68 & 21.0 & 15 & 30.3 & 276
\end{tabular}

$\operatorname{Im}[\tilde{\sigma}(\omega)]$, parts of the frequency-dependent complex conductivity. The complex conductivity is extracted from the measured differential transmitted field as described above. $\operatorname{Im}[\tilde{\sigma}(\omega)]$ is negative, and $\operatorname{Re}[\tilde{\sigma}(\omega)]$ decreases with decreasing frequency. This behavior is characteristic of a material with inhibited longrange transport.

The frequency-dependent complex conductivities of P3HT, $0.2,0.5$, and 0.8 films at $0.5,2,5$, and 75 ps after $500 \mathrm{~nm}$ excitation were measured, and the results are displayed in Figures $4 \mathrm{a}-\mathrm{d}$. The excitation density is $400 \mu \mathrm{J} / \mathrm{cm}^{2}$. At a pump delay of $0.5 \mathrm{ps}$, we observe a peak in $\operatorname{Re}[\tilde{\sigma}(\omega)]$ that occurs around $60 \mathrm{~cm}^{-1}$. This peak is less pronounced in the 0.5 and 0.8 films. Generally, the complex conductivity for all films at all the measured delay times exhibits significant $\operatorname{Im}[\tilde{\sigma}(\omega)]$ that is negative and decreases linearly with increasing frequency and a nonzero $\operatorname{Re}[\tilde{\sigma}(\omega)]$ that either increases with increasing frequency or is constant. On the basis of these results, we conclude that charge carriers are the main contributors to all of the measured data for pump delays greater than at least $0.5 \mathrm{ps}$, as will be discussed later in the context of two different models
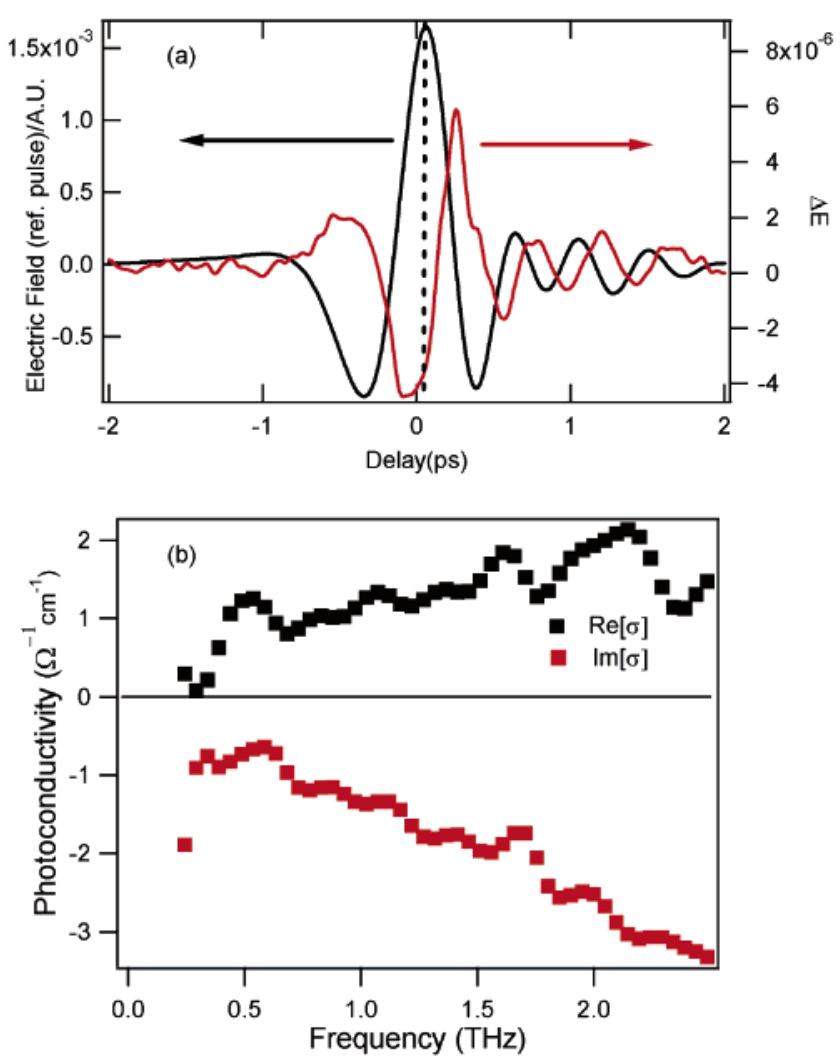

Figure 3. (a) Typical $\Delta E(t, \tau)$ (red) overlaid with a reference pulse (black), collected from the 0.5 film at 5 ps pump delay. The differential field has been multipled by 100 . The response is essentially the mirror image of the reference pulse but has been advanced slightly, indicative of a free carrier response. (b) $\operatorname{Re}[\tilde{\sigma}(\omega)]$ (black) and $\operatorname{Im}[\tilde{\sigma}(\omega)]$ (red) determined from the $\Delta E(t, \tau)$ and $E(t)$ traces of part a using the procedure described in the text.

that describe the frequency-dependent conductivity, the localized Drude and the Drude-Smith models. In addition, the expected exciton response, which is not implicitly included in these models, will be discussed.

Pump-Power-Dependent Dynamics. To further develop our understanding of the charge generation and recombination processes, we conducted pump-power-dependent terahertz measurements on each of the blended PCBM/P3HT film samples. Figure 5 shows the dynamics of the 0.5 film at four excitation densities $\left(315,420,530\right.$, and $\left.835 \mu \mathrm{J} / \mathrm{cm}^{2}\right)$. The dynamics at the two lowest excitation densities are similar, and the signal size is approximately proportional to the excitation density. The decay at early times becomes faster and more dominant with increasing pump power. In Figure 6 we show the terahertz signal values at 1 and 75 ps for $0.2,0.5$, and 0.8 films plotted as a function of excitation density. The data were fit by a powerlaw function, $y=a x^{k}$, and the exponents $(k)$ are summarized in Table 2.

\section{Analysis}

The frequency dependence in the terahertz regime may be used to distinguish between charge carriers and excitons. ${ }^{20}$ In general, $\operatorname{Re}[\tilde{\sigma}(\omega)]=\epsilon_{0} c \alpha(\omega) n(\omega)$, where $\alpha(\omega)$ is the photoinduced absorption coefficient (at $\omega$ ). For tightly bound excitons, terahertz radiation $\left(v<100 \mathrm{~cm}^{-1}\right)$ is far from any excited-state exciton absorption resonances; therefore, no absorption occurs by a photoexcited exciton, and $\operatorname{Re}[\tilde{\sigma}(\omega)]$ $=0$. The imaginary part of the conductivity is given by $\operatorname{Im}[\tilde{\sigma}(\omega)]=\left(\epsilon_{0} c^{2} \alpha(\omega)^{2} / 4 \omega\right)-\epsilon_{0} \omega \Delta \epsilon$, and when $\alpha(\omega)=0$, 

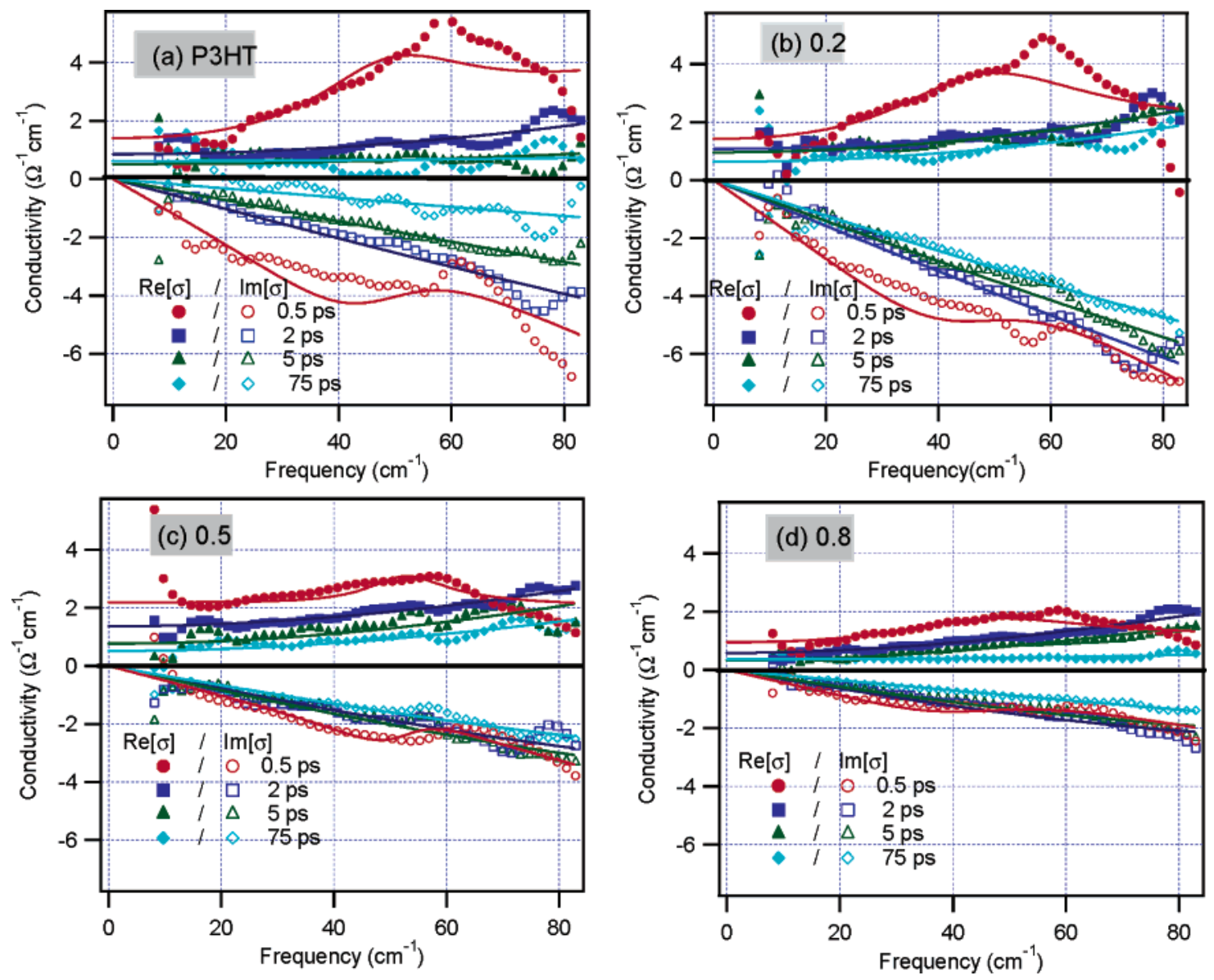

Figure 4. Complex conductivity of (a) P3HT, (b) 0.2 , (c) 0.5 , and (d) 0.8 films measured at 0.5 ps (circles), 2 ps (squares), 5 ps (triangle), and $75 \mathrm{ps}$ (diamond) after $500 \mathrm{~nm}$ pulse excitation. The excitation density is $400 \mu \mathrm{J} / \mathrm{cm}^{2}$. The lines are fits to the data using the DSM. The fitting procedure and results are discussed in the text.

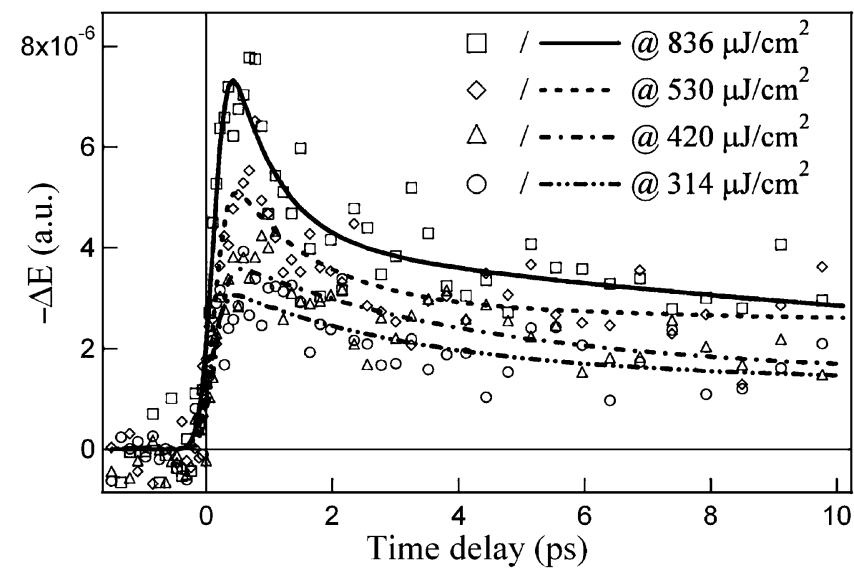

Figure 5. TRTS dynamic scans of the 0.5 film at four different excitation densities: $836 \mu \mathrm{J} / \mathrm{cm}^{2}$ (squares), $530 \mu \mathrm{J} / \mathrm{cm}^{2}$ (diamonds), 420 $\mu \mathrm{J} / \mathrm{cm}^{2}$ (triangles), and $314 \mu \mathrm{J} / \mathrm{cm}^{2}$ (circles). Lines are multiexponential fits as guides to the eye.

$\operatorname{Im}[\tilde{\sigma}(\omega)]=-\epsilon_{0} \omega \Delta \epsilon$, where $\Delta \epsilon$ is the change in the dielectric constant induced by the photoexcitation. Creation of an exciton modifies the microscopic polarizability, thus inducing changes in the macroscopic dielectric constant. The effect is described by the well-known Clausius-Mossotti relation, $\epsilon=(2 N \alpha$ $\left.+3 \epsilon_{0}\right) /\left(3 \epsilon_{0}-N \alpha\right)$, which relates the microscopic polarizability, $\alpha$, to the macroscopic dielectric constant $\epsilon$. Therefore, the signature of excitons with a large binding energy is an $\operatorname{Im}[\tilde{\sigma}(\omega)]$ that is negative with a straight line that passes through zero at zero frequency and $\operatorname{Re}[\tilde{\sigma}(\omega)]=0$. In contrast, free charge carriers will show significant induced absorption, yielding nonzero values of $\operatorname{Re}[\tilde{\sigma}(\omega)]$.

We now discuss two models of the conductivity that are based upon modifications to the Drude model: the localized Drude model (LDM) and the Drude-Smith model (DSM). The Drude model, operative in most bulk semiconductors, is the simplest model of conductivity and treats conduction electrons as free to move under the influence of an applied field. ${ }^{28}$ The model predicts a maximum value in $\operatorname{Re}[\tilde{\sigma}(\omega)]$ at zero frequency (or $\mathrm{dc})$. At "dc", $\omega=0, \operatorname{Im}[\tilde{\sigma}(\omega)]$ is zero and increases with increasing frequency to a maximum that occurs at the carrier scattering rate. For real materials the simple Drude model is inadequate and must be modified, ${ }^{22,28-30}$ but in general, $\tilde{\sigma}(\omega)$ has distinct features near the frequency corresponding to the carrier scattering rate. In contrast to the Drude model, we observe that $\operatorname{Re}[\tilde{\sigma}(\omega)]$ decreases with decreasing frequency (the data show a positive slope) and $\operatorname{Im}[\tilde{\sigma}(\omega)]$ is negative (Figure $4)$. These features cannot be reproduced with the conventional Drude model. A positive slope in $\operatorname{Re}[\tilde{\sigma}(\omega)]$ indicates that longrange transport is hindered and the conductivity measured in a terahertz experiment will be greater than that measured at dc, $\omega=0$.

Such non-Drude behavior in the complex conductivity has been observed in other studies utilizing terahertz spectroscopy ${ }^{17,31}$ and far-IR reflectivity. ${ }^{32,33}$ Jeon et al. ${ }^{31}$ studied doped conducting polymers using static terahertz time-domain spectroscopy (THz-TDS) and found that for doped polypyrrole the response could be characterized using a Drude treatment. In contrast, the response from doped poly(3-methylthiophene) differed drastically from that predicted by the Drude model. ${ }^{17}$ 

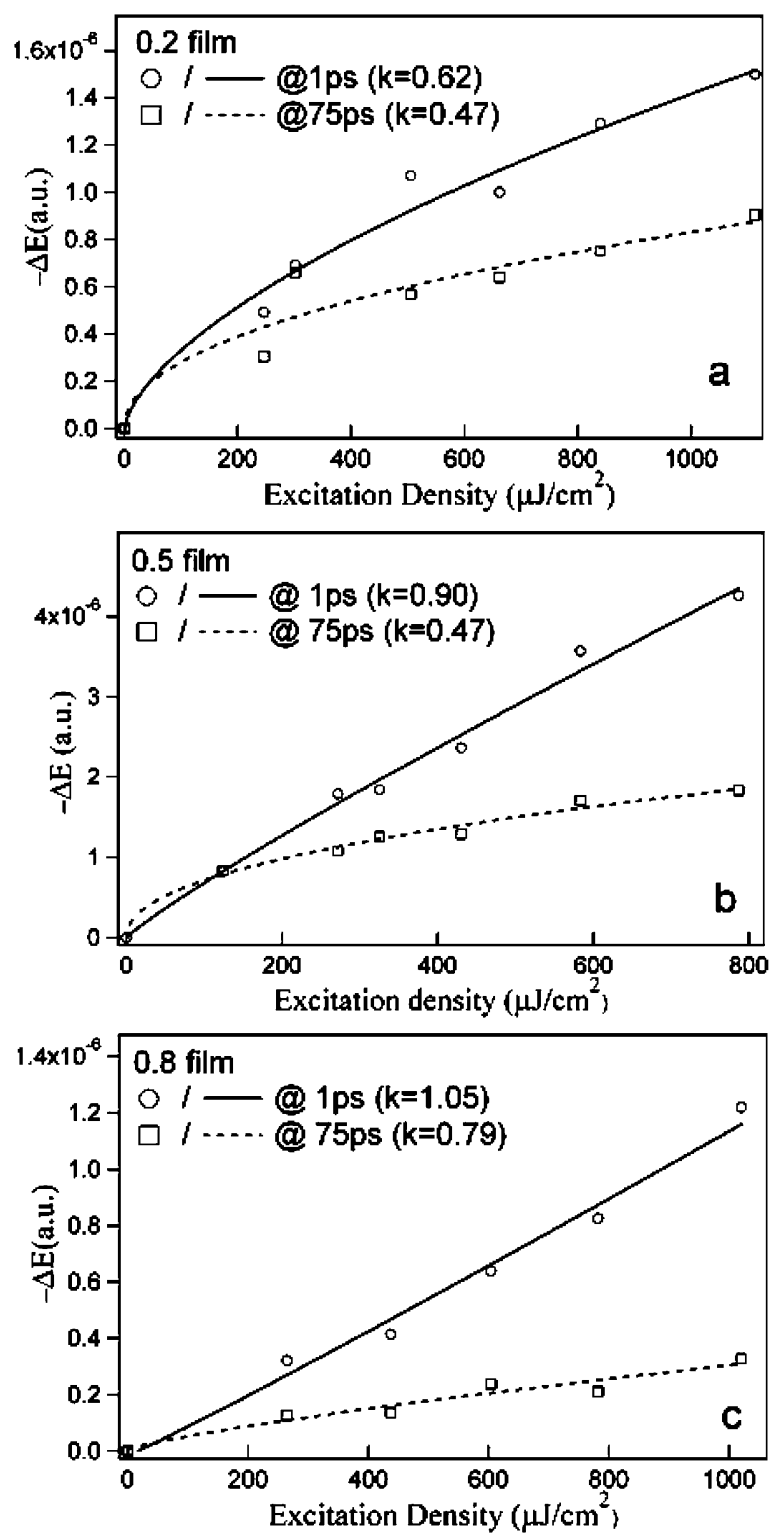

Figure 6. TRTS response as a function of excitation density collected at $1 \mathrm{ps}$ (open circles) and $75 \mathrm{ps}$ (open squares) for (a) 0.2, (b) 0.5 , and (c) 0.8 blended films. Lines are power-law $\left(y=a x^{k}\right)$ fits, and the exponents from fitting are summarized in Table 2.

TABLE 2: Exponent $k$ from Power-Law Fitting $\left(y=a x^{k}\right)$ of Pump-Power Dependence for 0.2, 0.5, and 0.8 Films

\begin{tabular}{cccc}
\hline & 0.2 film & 0.5 film & 0.8 film \\
\hline $1 \mathrm{ps}$ & 0.62 & 0.90 & 1.05 \\
$75 \mathrm{ps}$ & 0.47 & 0.47 & 0.79
\end{tabular}

They found that the LDM adequately represented the measured complex conductivity. In addition, the LDM has also been employed by Heeger and co-workers to describe low-frequency reflectivity data for conductive polymers. ${ }^{32}$

The LDM is a modified form of the Drude model valid for weak Anderson localization ${ }^{34}$ (when $l>a$, where $l$ is the mean free path and $a$ is the distance between molecular units, i.e., the unit cell length). However, we find that for photoexcited P3HT, $l \approx a$ (see below), and therefore the LDM does not describe photoconductivity in P3HT and specifically does not reproduce the frequency dependence of the $\operatorname{Im}[\tilde{\sigma}(\omega)]$. How ever, it will be useful to introduce and discuss this model for reference during the forthcoming analysis. The functional form of $\operatorname{Re}[\tilde{\sigma}(\omega)]$ for the LDM is given by

$$
\begin{array}{r}
\operatorname{Re}\left[\tilde{\sigma}_{\mathrm{LDM}}(\omega)\right]=\left[\frac{\epsilon_{0} \omega_{\mathrm{p}}{ }^{2} \tau}{1+\tau^{2} \omega^{2}}\right]\left[1-\frac{1}{\tau^{2}}\left(\frac{C}{\left(k_{\mathrm{f}} v_{\mathrm{f}}\right)^{2}}\right)+\frac{(3 \omega)^{1 / 2}}{\tau^{3 / 2}}\right. \\
\left.\left(\frac{C}{\left(k_{\mathrm{f}} v_{\mathrm{f}}\right)^{2}}\right)\right]
\end{array}
$$

where $\omega_{\mathrm{p}}$ is the plasmon frequency, $\tau$ is the carrier scattering time, $C$ is a constant with a value close to unity, $k_{\mathrm{f}}$ is the quasiFermi wavevector, and $v_{\mathrm{f}}$ is the quasi-Fermi velocity. The first term, $\epsilon_{0} \omega_{\mathrm{p}}{ }^{2} \tau /\left(1+\omega^{2} \tau^{2}\right)$, is the real part of the Drude conductivity where $\epsilon_{0} \omega_{\mathrm{p}}{ }^{2} \tau=e N \mu$. The imaginary part of the conductivity must be obtained via a Kramers - Kronig transformation. This model is characterized by a dc conductivity given by $\tilde{\sigma}_{\mathrm{LDM}}(0)$ $=e N \mu\left(1-C /\left(k_{\mathrm{f}} v_{\mathrm{f}} \tau\right)^{2}\right)$. Since the dc conductivity cannot physically be less than zero this imposes a restriction that $C /\left(k_{\mathrm{f}} v_{\mathrm{f}}\right)^{2}<\tau^{2}$. Thus, for transport to occur, $v_{\mathrm{f}} \tau>1 / k_{\mathrm{f}}$. Since $v_{\mathrm{f}} \tau$ is the mean free path $(l)$ and $1 / k_{\mathrm{f}}=a / \pi$, the restriction indicates that $l>a / \pi$; this relationship defines the well-known metal-insulator transition.

To account for materials with strong carrier localization due to nanoscale inhomogeneity and disorder, we now consider the DSM. Smith proposed a generalization of the Drude model that works well for semimetals near the metal-insulator transition, such as liquid $\mathrm{Hg}^{35}$ The functional form of the DSM is given by

$$
\tilde{\sigma}_{\mathrm{DSM}}(\omega)=\left[\frac{\epsilon_{0} \omega_{\mathrm{p}}{ }^{2} \tau}{1-\mathrm{i} \tau \omega}\right]\left[1+\sum_{n=1}^{\infty} \frac{c_{n}}{(1-\mathrm{i} \omega \tau)^{n}}\right]
$$

Smith suggested truncating the series at $n=1$, such that $c_{n}$ $=0$ for $n>1 ; c_{1}$ is the "persistence of velocity" and is allowed to be between -1 and 1 . The value is related to a "memory" effect during carrier scattering. In the Drude model, the carrier loses "memory" of its incoming velocity, and the carrier's momentum is randomized following each scattering event. However, in the DSM the carrier retains some fraction, $c_{1}$, of the incoming velocity. When $c_{1}$ is negative this indicates that the carrier undergoes back-scattering or localization. At dc the conductivity is given by $\tilde{\sigma}_{\mathrm{DSM}}(0)=e N \mu\left(1+c_{1}\right)$. Therefore, at $\omega=0$ we can equate the DSM and LDM models and find that $c_{1}=-C /\left(k_{\mathrm{f}} v_{\mathrm{f}} \tau\right)^{2}$; at higher frequencies the DSM and LDM models diverge. If $c_{1}$ is close to -1 , then $l \approx a$. The DSM can accommodate materials that are very close to the metalinsulator transition, whereas the LDM does not.

Figure 7 compares fitting with the LDM and DSM to the complex conductivity in the 0.2 film at a delay of 2 ps. Clearly the LDM cannot represent $\operatorname{Im}[\tilde{\sigma}(\omega)]$ but also fails to reproduce $\operatorname{Re}[\tilde{\sigma}(\omega)]$ adequately. The measured $\operatorname{Re}[\tilde{\sigma}(\omega)]$ has an $\omega^{2}$ dependence, indicative of a material on the insulator side of a metal-insulator transition, whereas the LDM has an $\omega^{1 / 3}$ dependence that can describe a material on the metal side of a metal-insulator transition. The results of fitting all data for delay times greater than $0.5 \mathrm{ps}$ by the DSM model are displayed as solid lines in Figure 4, and the extracted parameters are reported in Table 3. The carrier scattering time, $\tau$, and plasmon frequency are the parameters that are directly extracted from the fits. The mobility, $\mu$, and carrier density, $N$, can be obtained if the effective mass, $m^{*}$, of the carriers is known; $\mu=e \tau / m^{*}$, and $N$ $=\epsilon_{0} \omega_{\mathrm{p}}{ }^{2} m^{*} / e^{2}$. We take the average effective mass of the electron and hole in $\mathrm{P} 3 \mathrm{HT}$ to be $1.7,{ }^{17}$ noting that uncertainties in the effective mass lead directly to uncertainties in the extracted values of the carrier density and mobility. For 2 of the 16 cases shown in Table 3, the P3HT and the 0.8 films at 


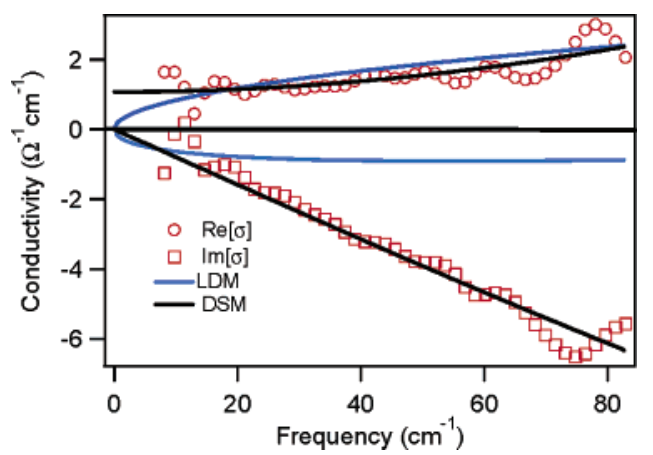

Figure 7. Comparison of fitting with the LDM and DSM to the complex conductivity in the 0.2 film at 2 ps pump delay.

TABLE 3: Results of the Application of a Drude-Smith-Based Analysis of Frequency-Dependent Conductivity Data Measured for the P3HT, 0.2, 0.5, and 0.8 Films at Probe Delays of 0.5, 2, 5, and 75 ps

\begin{tabular}{|c|c|c|c|c|c|}
\hline$\tau_{\mathrm{d}}(\mathrm{ps})$ & $\mu\left(\mathrm{cm}^{2} \mathrm{~V}^{-1} \mathrm{~s}^{-1}\right)$ & $N\left(\mathrm{~cm}^{-3}\right)$ & $c_{1}$ & $\Delta \epsilon$ & yielc \\
\hline \multicolumn{6}{|c|}{ P3HT } \\
\hline $\begin{array}{l}0.5 \\
2\end{array}$ & & & & & \\
\hline $\begin{array}{l}2 \\
5\end{array}$ & $\begin{array}{l}8(2) \\
8(2)\end{array}$ & $\begin{array}{l}2.8(0.1) \times 10^{19} \\
0.97(0.05) \times 10^{19}\end{array}$ & $\begin{array}{l}-0.97 \\
-0.96\end{array}$ & & $\begin{array}{r}24 \% \\
89\end{array}$ \\
\hline 75 & $8(4)$ & $0.4(0.2) \times 10^{19}$ & -0.954 & 1.11 & $4 \%$ \\
\hline \multicolumn{6}{|c|}{0.2 film } \\
\hline 0.5 & & & & & \\
\hline 2 & 8 & $4.1 \times 10^{19}$ & -0.983 & & $35^{\circ}$ \\
\hline 5 & 8 & $3.7 \times 10^{19}$ & -0.980 & & $33^{c}$ \\
\hline 75 & 8 & $3.2 \times 10^{19}$ & -0.985 & & $28 \%$ \\
\hline \multicolumn{6}{|c|}{0.5 film } \\
\hline 0.5 & & & & & \\
\hline 2 & 13.8 & $0.83 \times 10^{19}$ & -0.925 & & $14{ }^{\circ}$ \\
\hline 5 & 13.8 & $0.80 \times 10^{19}$ & -0.958 & & 13 \\
\hline 75 & 13.8 & $0.65 \times 10^{19}$ & -0.964 & & $10 \%$ \\
\hline \multicolumn{6}{|c|}{0.8 film } \\
\hline 0.5 & & & & & \\
\hline 2 & 18.5 & $0.37 \times 10^{19}$ & -0.947 & & 14 \\
\hline 5 & 18.5 & $0.32 \times 10^{19}$ & -0.964 & & 13 \\
\hline 75 & 18.5 & $0.06 \times 10^{19}$ & -0.8 & 0.9 & 30 \\
\hline
\end{tabular}

a delay of $75 \mathrm{ps}$ required an additional parameter for proper fitting, which represents an exciton response as discussed in the first paragraph of this section.

We find that the mobility increases from the P3HT to the 0.8 film. We cannot distinguish between electrons and holes in these measurements; only the sum of the mobilities can be extracted. As the percentage of PCBM increases, aggregates of PCBM form, and the increasing mobility may therefore be due to an increasing electron mobility within these aggregates. Interestingly, the average mobilities are consistent with a volume-weighted sum of a mobility in PCBM and P3HT with $\mu_{\mathrm{PCBM}}=22.6$ and $\mu_{\mathrm{P} 3 \mathrm{HT}}=8$. (The volume fraction of $\mathrm{P} 3 \mathrm{HT}$ in the 0.5 film is 0.6.) The mobility extracted from terahertz measurement reflects transport on a small length scale, as the terahertz field induces a small perturbation on the motion of the carriers on a subangstrom scale, and thus long-range transport does not contribute significantly to the measured mobility. A predicted dc mobility can be obtained by multiplying the "ac" mobility by $\left(1+c_{1}\right)$. A value of $c_{1}$ close to -1 suggests that the carriers are localized. To better understand this parameter we use the relation between the DSM and the LDM, $c_{1}=-C /\left(k_{\mathrm{f}} v_{\mathrm{f}} \tau\right)^{2}$. For the 0.2 film, the carrier scattering time, $\tau$, is $8 \mathrm{fs}$, and $c_{1}$ is -0.98 ; therefore, $C /\left(k_{\mathrm{f}} v_{\mathrm{f}}\right)^{2}=6.3 \times 10^{-29} \mathrm{~s}^{2}$. Assuming that $a \approx 0.5 \mathrm{~nm}$, the quasi-Fermi velocity is $2.0 \times$ $10^{6} \mathrm{~cm} \mathrm{~s}^{-1}$ and yields a mean free path of $0.16 \mathrm{~nm}$, indicating that the conduction band in P3HT consists of highly localized states with poor transport properties.
The photon-to-carrier yield ( $\left.N / N_{\text {photoexcitation }}\right)$ is listed in Table 3 and varies for both the probe delay and the value of $W_{\text {РСBM. }}$. For example, at $2 \mathrm{ps}$ after the pump, the yield for the P3HT film is $24 \%$, that for the 0.2 film is $35 \%$, and the 0.5 and 0.8 films show yields of $14 \%$; while at 75 ps the yields for the P3HT and 0.8 films drops to $4 \%$ and $3 \%$ respectively, the 0.2 film retains the highest yield of $28 \%$, and the 0.5 film follows with a yield of $10 \%$. Although the reliability of the absolute values of the yields depends on $m^{*}$ and therefore could be off by an order of magnitude, if $m^{*}=1.0$, then, for example, the yield at $2 \mathrm{ps}$ for the 0.2 film would be $22 \%$. The effect of $m^{*}$ cancels out when comparing the relative yields among samples. There is also an additional uncertainty with the absolute value of the reported quantum yields associated with the photons absorbed; we have assumed that $80 \%$ of the input photons are absorbed by the sample.

The frequency-dependent conductivity at a delay time of 0.5 ps displays interesting behavior that cannot be modeled with the LDM or DSM. The species assumed to be present at this delay time consist of charge carriers, bound polaron pairs, and excitons, as discussed later. Bound polaron pairs will have a very weak binding energy and therefore a resonance in the terahertz frequency window. We included a Lorenztian term in the conductivity given by

$$
\tilde{\sigma}_{\text {Lor }}(\omega)=\frac{\mathrm{i} \epsilon_{0} \omega G}{\left(\omega_{0}^{2}-2 \mathrm{i} \omega \gamma-\omega_{\mathrm{f}}^{2}\right)}
$$

where $G$ is a coupling parameter that is a product of the density of bound polaron pairs and their terahertz frequency cross section, $\omega_{0}$ is the resonance frequency, and $\gamma$ is its line width. We find that summing this term with the DSM enables fitting of the observed data. For all samples, $\omega_{0}=51.0 \times 2 \pi \mathrm{cm}^{-1}$, and $\gamma=21 \times 2 \pi \mathrm{cm}^{-1}$. Therefore, we conclude that bound polaron pairs are a precursor to charge carriers in the polymer charge generation process and that at $0.5 \mathrm{ps}$ a mixture of charge carriers and bound polaron pairs exists. However, the frequencydependent data do not contain sufficient information to determine the ratio of bound polaron pairs to charge carriers.

\section{Discussion}

We now discuss the photoinduced charge separation processes in our samples. In the pure polymer film, P3HT molecules are excited above the absorption gap $(\sim 1.9 \mathrm{eV})$ by using $500 \mathrm{~nm}$ light $(\sim 2.5 \mathrm{eV})$ to generate excitons - tightly bound electronhole pairs on a single polymer chain. In composite samples, PCBM may also absorb photons at $500 \mathrm{~nm}$ (Figure 1). However, on the basis of a published estimation, ${ }^{13}$ even for $80 \%$ PCBM in the blended film, approximately two-thirds of the photons absorbed by the mixture are due to absorption by the polymer. We therefore assume that $500 \mathrm{~nm}$ pump pulses primarily excite P3HT molecules, thus producing excitons. The lowest unoccupied molecular orbital (LUMO) of PCBM is lower than the LUMO of P3HT, ${ }^{3}$ so photoexcited P3HT molecules close to a PCBM interface will transfer electrons to PCBM leaving holes on the polymer chains, possibly through charge-transfer intermediates. Figure $8 \mathrm{a}$ is a schematic of the possible pathways that an initial photoexcitation may proceed to produce longlived carriers. We propose three types of excitons that may be produced, labeled 1, 2, and 3 in Figure 8. These excitons only differ by their relative fates; type 1 excitons produce charge carriers in isolated P3HT domains via an interchain chargetransfer process that produces bound polaron pairs, where the electron-hole pairs reside on neighboring chains and are bound 


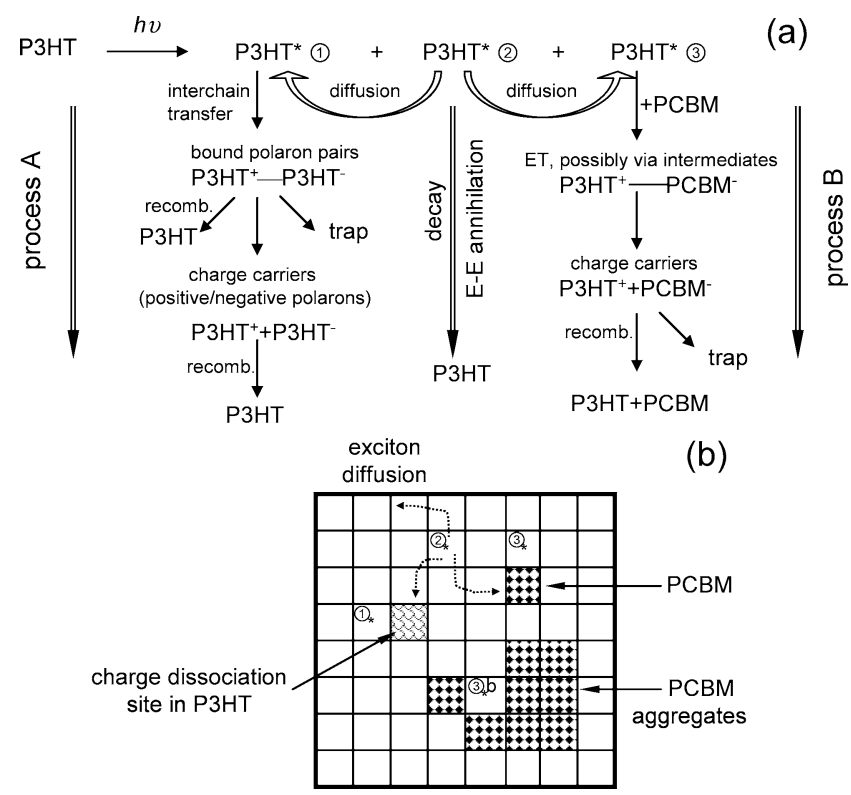

Figure 8. Schematic of the interfacial and interchain charge carrier separation process. (a) Different fates of the three exciton species: 1, 2, and 3. Process A, with type 1 close to charge dissociation sites in $\mathrm{P} 3 \mathrm{HT}$, is an interchain charge-transfer process that proceeds through bound polaron pairs to produce long-lived charge carriers. Process A occurs in isolated domains of P3HT, as shown in part b. Process B is an interfacial charge-transfer process that occurs at the P3HT/PCBM interface. In the blended films, processes A and B compete. Type 2 excitons may diffuse into process A or B or decay via nonradiative or radiative processes. Photoexcitation creates a random distribution of type 1,2, and 3 excitons, shown in part b. Type $3 b$ excitons differ from type 3 excitons by the presence of PCBM aggregates. The formation of these aggregates leads to an effective decrease in the photon-to-carrier yield.

by a Coulomb potential, ${ }^{36}$ which dissociate creating charge carriers, labeled process A in Figure 8a; type 3 excitons produce charge carriers via an interfacial charge transfer at a PCBM/ $\mathrm{P} 3 \mathrm{HT}$ interface, process $\mathrm{B}$; type 2 excitons either diffuse to a PCBM interface to undergo process $\mathrm{B}$, undergo interchain charge transfer, process A, or decay via a nonradiative or radiative process. Photoexcitation produces a random distribution of type 1, 2, and 3 excitons, as shown schematically in Figure 8b, with differing amounts of each species depending on the PCBM concentration. We also distinguish between type 3 and $3 b$ excitons, where type $3 b$ excitons are near a PCBM aggregate, while type 3 excitons are near smaller or isolated PCBM moieties; these will be discussed in the context of the relative photon-to-charge carrier yields.

In pure P3HT only type 1 and 2 excitons exist, and thus, no process $\mathrm{B}$ is operable. Type 1 excitons, those close to a charge dissociation center, which may be another polymer chain, undergo interchain charge transfer to produce bound polaron pairs. The bound polaron pairs can migrate along the conjugated polymer chains, dissociate to form free charge carriers, or simply recombine. Fast recombination of bound polaron pairs, which proceeds via an intrinsic or extrinsic process (defect trapping), competes with the generation of charge carriers. Charge carriers that survive the competition can delocalize along the polymer chains, avoid rapid recombination and/or trapping, and produce long-lived charge carriers. The production of long-lived charge carriers in the P3HT film occurs with very low probability, only $\sim 3 \%$ at 75 ps in our measurements. Those excitons, type 2, that are far from charge dissociation sites may diffuse to a dissociation site and produce a charge carrier, meet another exciton, and undergo exciton-exciton annihilation or simply decay to the ground state. However, exciton diffusion is slow on the time scale of these measurements, so for these experiments type 1,2, and 3 excitons are effectively stationary.

Charge carriers contribute significantly to the measured photoconductivity for all the detected delay times in pure P3HT (see above). However, at $0.5 \mathrm{ps}$, an additional term representing a contribution from bound polaron pairs is included to obtain reasonable fitting, though it is difficult to determine the relative yield of bound polaron pairs compared to that of charge carriers. Therefore, the instantaneous rise in the photoconductivity (Figure 2) is assigned to bound polaron formation, resulting from interchain charge transfer. The formation of bound polaron pairs in polythiophene films on a sub-picosecond time scale has been previously measured by transient absorption. ${ }^{37}$ The fast (subpicosecond) decay may result from bound polaron recombination, trapping by defect states, and/or dissociating into charge carriers (positive/negative polarons). After this initial fast decay, the small long-lived signal is assigned to charge carriers that are delocalized along the polymer chain; this signal slowly decays due to recombination of delocalized polarons. Similar transient absorption dynamics with fast and slow decay components from pure poly(3-octylthiophene) (P3OT) film have been reported. ${ }^{15}$

At $75 \mathrm{ps}$, a parameter representing an exciton response is necessary for fitting the frequency-dependent photoconductivity of P3HT. Excitons contribute to the measured photoconductivity at a much reduced level in comparison to charge carriers; therefore, a significant population of charge carriers will obscure any exciton contribution. At later delay times ( $75 \mathrm{ps}$ ) when the charge carrier concentration decreases significantly the exciton response emerges. Previous studies have shown that excitons in P3HT thin films decay nonexponentially with decay constants ranging from 140 to $790 \mathrm{ps}^{38}$ Therefore, within the $150 \mathrm{ps}$ of our measurement, type 2 excitons are always present, but we only detect them when the charge carriers have decayed.

Hendry et al. ${ }^{20}$ measured carrier generation in MEH-PPV films by the same terahertz method as we use here. They find that at $10 \mathrm{ps}$ the extracted conductivity is mostly imaginary and therefore arises from excitons. ${ }^{20}$ In contrast, at $10 \mathrm{ps}$ in P3HT films a significant population of charge carriers remains. The difference may originate from the different structures of regioregular P3HT and MEH-PPV. The highly regioregular poly(3-alkylthiophene)s have an extended conjugation length, self-organized structure, and self-assembly crystalline texture due to less steric hindrance in comparison to the regiorandom counterpart. ${ }^{39}$ As the regioregularity of P3HT increases, a gradual red shift of the absorption spectra is observed, ${ }^{40}$ which can be attributed to an increased conjugation length due to increased ordering. X-ray diffraction analysis has also shown that cast films of regioregular P3HT form a self-organized lamellar morphology with three-dimensional ordering of the polymer chains. ${ }^{40}$ Therefore, the extended conjugation length and reduced interchain distance between the polythiophene backbones in regioregular P3HT are expected to increase the possibility for interchain charge transfer upon photoexcitation compared to MEH-PPV, which does not order as well as P3HT. The extended conjugation length from the regiospecificity also facilitates charge carrier delocalization along the conjugated P3HT polymer chains, thereby reduces recombination and trapping, and enhances the lifetime of the charge carriers.

Charge carriers from both interchain transfer within the polymer regions and interfacial charge transfer with PCBM contribute to the photoconductivity, and thus the photoconductivity dynamics of the blended films contains contributions from 
both processes $\mathrm{A}$ and $\mathrm{B}$ (Figure 8). In the composite films photoexcitation creates excitons of type 1,2, and 3 with different relative ratios depending on the amount of PCBM, and therefore, process $\mathrm{A}$ competes with process $\mathrm{B}$ depending on the amount of net P3HT content in the mixture. Differences in the dynamical response among the different films result from this competition. We observe a response from bound polaron pairs in all of the films, but its contribution decreases from the P3HT to 0.8 films consistent with increasing type 3 excitons and decreasing type 1 excitons. Additionally, we note that our measurements do not distinguish whether bound and/or free polarons from process A diffuse along a polymer chain to a PCBM and then transfer charge. However, if this process does occur it must occur either very fast (within $\sim 1 \mathrm{ps}$ ) or with a very small yield because otherwise this would appear as a slow risetime in the detected signal.

For the 0.2 film, the photoconductive dynamics are quite different from those in P3HT due to the interfacial charge transfer (process B). The photoconductivity at $\tau_{\mathrm{d}}=0$ contains contributions from both bound polaron pairs (interchain charge transfer) and charge carriers (interfacial charge transfer). The decay after the initial rise is slower and has a smaller amplitude than that of P3HT, indicating a decreased bound polaron population from interchain charge transfer. After the initial decay, the photoconductivity displays a long-lived signal decaying with a time constant of $\sim 500 \mathrm{ps}$. Since this component is absent from the P3HT film and the charge carrier yield from interchain interaction of pure P3HT is very low (only $3 \%$ at 75 $\mathrm{ps}$ ), it is assigned to the charge carriers from interfacial charge transfer (process B) with a longer decay time constant.

The 0.5 film shows similar photoconductivity dynamics as the 0.2 film, except that the initial decay component is slightly slower and the long-lived component decays slightly faster, and the relative yield is lower than that of the 0.2 film. The comparison of relative yield among all samples will be discussed later. In the 0.5 film both processes A and B contribute to the detected signal; therefore, a slower initial decay indicates less of process A (or less of the type 1 exciton) in the 0.5 film than the 0.2 film. The dynamics for $\tau_{\mathrm{d}} \gtrsim 20 \mathrm{ps}$ are roughly similar for the 0.2 and 0.5 films, suggesting a similar recombination process. However, the long-lived component of the 0.5 film decays a little faster than that of the 0.2 film; in the 0.5 film more PCBM molecules result in more aggregates, leading to more type $3 \mathrm{~b}$ excitons (close to PCBM aggregates) and less type 3 excitons in comparison to the 0.2 film.

For the $0.8 \mathrm{film}$, the polymer chains are quite isolated and disordered as indicated by the shift in the absorption spectrum; therefore the amount of type 1 exciton is very small, and process A is likely not a significant contributor to the dynamics. As a result, the initial rise of the photoconductivity signal closely represents the electron-transfer rate from P3HT to PCBM. The rate is $>0.4 \mathrm{ps}^{-1}$, limited by the instrument response, and consistent with previous observation, a $<0.3$ ps electron-transfer time measured from P3OT to $\mathrm{C}_{60}$ by phtoinduced absorption spectroscopy. ${ }^{15}$ In addition, as the amount of PCBM increases, large aggregates form, ${ }^{2}$ thus even more type $3 \mathrm{~b}$ and less type 3 excitons in the 0.8 film.

Comparing the pump-power-dependent measurements for the different films provides additional information. First, we compare the pump-power-dependent measurement at 1 ps. For the 0.2 film, the exponent $(k)$ for the $1 \mathrm{ps}$ response from the power-law fitting is 0.62 , while it is 0.9 for the 0.5 film and 1 for the 0.8 film. At early times for an increasing pump fluence, we observe a sublinear response in the production of charge carriers; this is consistent with exciton-exciton annihilation (EEA). Though we do not detect the exciton signal directly, EEA decreases the effective excitation density and hence diminishes interchain and/or interfacial charge separation. In contrast to the 0.2 film, the 0.5 and 0.8 films display a linear or near-linear pump power response; therefore, interfacial chargetransfer competes with EEA, because adding PCBM molecules increases the chain-to-chain distance, which reduces exciton concentration of types 1 and 2 and thus EEA.

The pump-power-dependent measurements at later delay times correspond to the recombination dynamics. The exponent from power-law fitting of the 0.2 and 0.5 films at $75 \mathrm{ps}$ is 0.47 . We assign this process to a second-order recombination process, e.g., recombination of an electron in PCBM and a hole in P3HT. This process is likely even slower than 500 ps; the uncertainty in this number is limited by our $\sim 150$ ps detection window. The decay dynamics of the 0.8 film shows a sublinear response at $75(k=0.79)$, which is mainly assigned to the contribution from electron trapping within PCBM aggregates with a faster decay rate than the second-order recombination process. Even though there may exist a small amount of recombination between charged polymer and PCBM molecules, this process will not be significant because the transferred electron may migrate into PCBM aggregates and be further away from the hole in P3HT.

In addition to the dynamics, comparing the relative yields among the samples sheds light on how the composition affects device performance. $\mathrm{P} 3 \mathrm{HT}$ has a relatively high photon-tocarrier yield $(\sim 19 \%)$ at $2 \mathrm{ps}$, indicating a high concentration of type 1 excitons that produce charge carriers via interchain charge transfer in the pure polymer film (process A). However, the charge density decays very fast so that only $3 \%$ remains at 75 ps. Adding 20\% PCBM into the mixture leads to an increased concentration of type 3 excitons; the yield increases at both early and late delay times, indicating effective interfacial charge separation that enhances the carrier density and slows down the recombination. Surprisingly the yield of the 0.5 film is lower than that of the 0.2 film. As the PCBM concentration increases, larger PCBM aggregates form, which in turn decreases the interfacial surface area and thereby decreases the population of type 3 excitons and increases the population of type $3 \mathrm{~b}$ excitons. Given that the type 1,2, and 3 excitons are static on these time scales, as exciton diffusion is slow, a higher yield for the 0.2 film results from a larger population of type 3 versus type $3 b$ excitons. This may initially be considered a surprising result given the fact PCBM/P3HT photovoltaic devices perform better with increasing PCBM concentrations up to $W_{\mathrm{PCBM}}=0.5$. However, the device performance depends on many other longer-range charge transport processes. The increase in the PCBM aggregates leads to an increase in long-range charge transport. The 0.5 film maintains enough ordering in polymer chains to facilitate hole transfer along the polymer backbone and, meanwhile, with enough PCBM molecules and/or aggregates forms better contact with the electrode for efficient electron extraction. The 0.8 film has a similar yield at 2 ps to that of the 0.5 film but decays quickly to $2 \%$ at 75 ps due to trapping within PCBM aggregates, which is consistent with decreased device performance at a high PCBM weight ratio.

\section{Summary}

We utilized time-resolved terahertz spectroscopy with subpicosecond to nanosecond temporal resolution to investigate the photoinduced charge carrier generation dynamics of P3HT/ PCBM blended films with PCBM weight fractions of $0,0.2$, 
0.5 , and 0.8 . The frequency-dependent complex photoconductivity of these samples is measured and analyzed by the DSM to determine the charge carrier yield and average charge carrier mobility. Surprisingly, the 0.2 film shows a higher photon-tocarrier conversion yield at 75 ps than that of 0.5 film, while PCBM/P3HT photovoltaic devices with $W_{\mathrm{PCBM}}=0.5$ tend to perform better. The relatively lower yield of the 0.5 film at 75 ps is ascribed to more PCBM aggregates, which decrease effective interfacial charge transfer but facilitate efficient electron extraction in devices. The photoconductivity dynamics of all blended samples are compared and discussed based on a competition/combination between interchain charge separation (process A) and interfacial electron transfer (process B). The contribution from both processes is analyzed qualitatively with the help of excitation-density-dependent measurements. We find that at early delay ( $1 \mathrm{ps}$ ) the 0.2 film shows more interchain interaction than the 0.5 and 0.8 films, while at 75 ps the decay from the 0.2 and 0.5 films mainly results from second-order recombination between electrons in PCBM and holes in P3HT, and that for the 0.8 film the decay is due to electron trapping within PCBM aggregates.

Acknowledgment. The work is funded by the Photochemistry and Radiation Research program in the Office of Science, Office of Basic Energy Sciences, Division of Chemical Sciences, Geosciences and Biosciences, Department of Energy, under Contract No. DE-AC36-99GO10337 to the National Renewable Energy Laboratory (NREL). Additional support is also provided by the NREL Director's Discretionary Research and Development fund.

\section{References and Notes}

(1) Al-Ibrahim, M.; Roth, H. K.; Zhokhavets, U.; Gobsch, G.; Sensfuss, S. Sol. Energy Mater. Sol. Cells 2005, 85, 13.

(2) Chirvase, D.; Parisi, J.; Hummelen, J. C.; Dyakonov, V. Nanotechnology 2004, 15, 1317.

(3) Kim, Y.; Choulis, S. A.; Nelson, J.; Bradley, D. D. C.; Cook, S.; Durrant, J. R. J. Mater. Sci. 2005, 40, 1371.

(4) Kim, Y.; Choulis, S. A.; Nelson, J.; Bradley, D. D. C.; Cook, S.; Durrant, J. R. Appl. Phys. Lett. 2005, 86, 063502.

(5) Li, G.; Shrotriya, V.; Huang, J. S.; Yao, Y.; Moriarty, T.; Emery, K.; Yang, Y. Nat. Mater. 2005, 4, 864.

(6) Kim, J. Y.; Kim, S. H.; Lee, H. H.; Lee, K.; Ma, W. L.; Gong, X.; Heeger, A. J. Adv. Mater. 2006, 18, 572.

(7) Reyes-Reyes, M.; Kim, K.; Carroll, D. L. Appl. Phys. Lett. 2005, $87,3506$.

(8) Kim, Y.; Cook, S.; Tuladhar, S. M.; Choulis, S. A.; Nelson, J.; Durrant, J. R.; Bradley, D. D. C.; Giles, M.; McCulloch, I.; Ha, C. S.; Ree, M. Nat. Mater. 2006, 5, 197.
(9) Shrotriya, V.; Ouyang, J.; Tseng, R. J.; Li, G.; Yang, Y. Chem. Phys. Lett. 2005, 411, 138

(10) Al-Ibrahim, M.; Ambacher, O.; Sensfuss, S.; Gobsch, G. Appl. Phys. Lett. 2005, 86, 201120.

(11) Li, G.; Shrotriya, V.; Yao, Y.; Yang, Y. J. Appl. Phys. 2005, 98, 043704 .

(12) Huang, J. S.; Li, G.; Yang, Y. Appl. Phys. Lett. 2005, 87, 112105.

(13) Savenije, T. J.; Kroeze, J. E.; Wienk, M. M.; Kroon, J. M.; Warman, J. M. Phys. Rev. B 2004, 69, 155205.

(14) Lee, K.; Miller, E. K.; Sariciftci, N. S.; Hummelen, J. C.; Wudl, F.; Heeger, A. J. Phys. Rev. B 1996, 54, 10525.

(15) Kraabel, B.; McBranch, D.; Sariciftci, N. S.; Moses, D.; Heeger, A. J. Phys. Rev. B 1994, 50, 18543.

(16) Beard, M. C.; Turner, G. M.; Schmuttenmaer, C. A. J. Phys. Chem. $B$ 2002, 106, 7146

(17) Jeon, T. I.; Grischkowsky, D.; Mukherjee, A. K.; Menon, R. Appl. Phys. Lett. 2001, 79, 4142.

(18) Jeon, T. I.; Grischkowsky, D.; Mukherjee, A. K.; Menon, R. Appl. Phys. Lett. 2000, 77, 2452.

(19) Hendry, E.; Koeberg, M.; Schins, J. M.; Siebbeles, L. D. A.; Bonn, M. Phys. Rev. B 2004, 70, 033202.

(20) Hendry, E.; Koeberg, M.; Schins, J. M.; Nienhuys, H. K.; Sundstrom, V.; Siebbeles, L. D. A.; Bonn, A. Phys. Rev. B 2005, 71, 125201

(21) Hendry, E.; Schins, J. M.; Candeias, L. P.; Siebbeles, L. D. A.; Bonn, M. Phys. Rev. Lett. 2004, 92, 196601.

(22) Beard, M. C.; Turner, G. M.; Schmuttenmaer, C. A. Phys. Rev. B 2000, 62, 15764 .

(23) Wu, Q.; Litz, M.; Zhang, X. C. Appl. Phys. Lett. 1996, 68, 2924.

(24) Wu, Q.; Zhang, X. C. Appl. Phys. Lett. 1995, 67, 3523.

(25) Beaurepaire, E.; Turner, G. M.; Harrel, S. M.; Beard, M. C.; Bigot, J. Y.; Schmuttenmaer, C. A. Appl. Phys. Lett. 2004, 84, 3465.

(26) Beard, M. C.; Turner, G. M.; Schmuttenmaer, C. A. J. Appl. Phys. 2001, 90, 5915 .

(27) Schall, M.; Jepsen, P. U. Opt. Lett. 2000, 25, 13.

(28) Ashcroft, N. W. a. M., N.D. Solid State Physics; Harcourt Brace College Publishers: New York, 1976.

(29) Jeon, T. I.; Grischkowsky, D. Appl. Phys. Lett. 1998, 72, 2259.

(30) Katzenellenbogen, N.; Grischkowsky, D. Appl. Phys. Lett. 1992, 61,840 .

(31) Jeon, T. I.; Grischkowsky, D.; Mukherjee, A. K.; Menon, R. Synth. Met. 2003, 135, 451 .

(32) Lee, K. H.; Heeger, A. J.; Cao, Y. Phys. Rev. B 1993, 48, 14884.

(33) Tzamalis, G.; Zaidi, N. A.; Homes, C. C.; Monkman, A. P. Phys Rev. B 2002, 66, 085202 .

(34) Mott, N. F. Adv. Phys. 1985, 34, 329

(35) Smith, N. V. Physical Review B 2001, 64, 155106.

(36) Sakamoto, A.; Nakamura, O.; Yoshimoto, G.; Tasumi, M. J. Phys. Chem. A 2000, 104, 4198.

(37) Ruseckas, A.; Theander, M.; Andersson, M. R.; Svensson, M.; Prato, M.; Inganas, O.; Sundstrom, V. Chem. Phys. Lett. 2000, 322, 136.

(38) Magnani, L.; Rumbles, G. Unpublished results.

(39) Chan, H. S. O.; Ng, S. C. Prog. Polym. Sci. 1998, 23, 1167.

(40) Chen, T. A.; Wu, X. M.; Rieke, R. D. J. Am. Chem. Soc. 1995, 117,233 